\begin{abstract}
Center for Mathematical Studies in Economics and Management Science Northwestern University, Evanston, Illinois 60208
\end{abstract}

Discussion Paper No. 1063

\begin{abstract}
AXIOMATIC DERIVATION OF SCORING RULES
WITHOUT THE ORDERING ASSUMPTION
\end{abstract}

by

Roger B. Myerson ${ }^{*}$

September 1993

Abstract. Earlier derivations of scoring rules, by Smith [1973] and Young [1975], assumed that a voter can express only a rank ordering of the alternatives on his or her ballot. This paper shows that scoring rules can be derived without this ordering assumption. It is shown that a voting rule must be a scoring rule if it satisfies three basic axioms: reinforcement, overwhelming majorities, and neutrality. Other range and nonreversal axioms are also discussed.

Acknowledgements. In writing this paper, I have also benefitted from many discussions with Matthew Jackson. Support from the National Science Foundation grant SES-9308139 is also gratefully acknowledged.

* Department of Managerial Economics and Decision Sciences, J. L. Kellogg Graduate School of Management, Northwestern University, Evanston, IL 60208. 


\section{AXIOMATIC DERIVATION OF SCORING RULES WITHOUT THE ORDERING ASSUMPTION}

by Roger B. Myerson

\section{Introduction}

A scoring rule is a social choice procedure in which each individual submits a ballot that assigns some number of points to each of the various alternatives, and a social choice is selected from among the set of alternatives that get the maximum total points from all the individuals who vote. Scoring rules differ according to what is the set of vote vectors that individuals are allowed to write on their ballots. Under plurality voting, a voter can assign 1 point to only one alternative, and must assign 0 points to all other alternatives. Under approval voting, a voter can assign 1 or 0 points to each alternative independently, with no restriction on how many alternatives can be given a point on a voter's ballot. Under Borda voting, if $K$ denotes the set of available alternatives, then a voter must assign each of the numbers $(0,1, \ldots, \mathrm{K}-1)$ to one of the available alternatives.

The prominence of scoring rules, in both the theory and practice of voting, suggests that there may be some fundamental properties that distinguish scoring rules as a particularly good class of social choice procedures. This insight was partially confirmed by the work of Smith [1973] and Young [1975], who present axiomatic derivations of scoring rules. In their derivations, however, both Smith and Young assumed that individuals can vote only by expressing a rank ordering of the alternatives. That is, in Smith's and Young's formulations, the set of possible votes is required to be equal to the set of possible rank orderings of the alternatives.

To see that this ordering assumption is seriously restrictive, notice that 
it would exclude approval voting. Two voters who have the same preference ordering over a set of three alternatives could sincerely cast different votes under approval voting. One voter might vote for only their top-ranked alternative, while the other voter might vote for both their top-ranked and second-ranked alternatives. Indeed, these two voters might strictly prefer to cast such different votes in a randomized equilibrium of an approval-voting game, if they have different von Neumann-Morgenstern utility functions over the alternatives. (For example, consider a situation where the first voter has utilities ( $1, .1,0)$ for the three alternatives, the other voter has utilities $(1, .9,0)$, and any pair of alternatives is equally likely to be in close race that could be affected by one vote.) Thus, there is loss of generality in assuming that a voter can only express a rank ordering of the alternatives on his or her ballot.

This paper shows that Smith's and Young's results can be extended by dropping the assumption that votes are rank orderings. We impose here no assumptions about the structure of the set of permissible votes, except that it is some nonempty finite set. Smith [1973] assumed also that the outcome of the voting must determine a complete rank ordering of the alternatives. Young [1975] dropped this assumption and specified that a voting rule only needs to choose a nonempty subset of the alternatives; we follow Young in this regard.

So the voting rules that we consider here are all those that can be described in the following general terms. Let $\mathrm{K}$ be a nonempty finite set which denotes the set of available alternatives among which society must choose. Let $\mathrm{V}$ be another nonempty finite set which denotes the set of possible votes that any individual voter can specify on his or her ballot. We assume that individuals submit their ballots anonymously, so the result of any election can 
be described by a distribution that specifies how many of each kind of ballot have been submitted. Thus, the set of possible election results is the set of possible functions from $V$ into the nonnegative integers. This set of functions is here denoted by $z_{+}^{V}$. That is, the result of an election is a vector $\alpha=(\alpha(\mathrm{v}))_{\mathrm{v} \in \mathrm{V}}$ in $\mathrm{Z}_{+}^{\mathrm{V}}$, where, for each $\mathrm{v}$ in $\mathrm{V}, \alpha(\mathrm{v})$ denotes the number of voters who have chosen to cast the vote $\mathrm{v}$.

We will consider neutral voting procedures which treat the alternatives symmetrically, so we must admit the possibility of ties among alternatives. Thus, the outcome of the voting rule must be a nonempty subset of alternatives, which may include two or more alternatives, in the case of a tie. So a voting $\underline{\text { rule }}$ is formally defined to be a correspondence $F: \mathrm{Z}_{+}^{\mathrm{V}} \rightarrow \mathrm{K}$ such that

$$
\varnothing \neq \mathrm{F}(\alpha) \subseteq \mathrm{K}, \quad \forall \alpha \in \mathrm{Z}_{+}^{\mathrm{V}} .
$$

For any distribution $\alpha$ in $\mathrm{Z}_{+}^{\mathrm{V}}, \mathrm{F}(\alpha)$ denotes the choice set, or the set of winning alternatives, when $\alpha$ is the distribution of votes. That is, $i$ is in the set $F(\alpha)$ iff $i$ would be an admissible social choice after an election in which each vote $\mathrm{v}$ in $\mathrm{V}$ was cast by $\alpha(\mathrm{v})$ voters. If there is no tie and alternative $i$ would be the unique winner of the election when distribution $\alpha$ is cast by the voters, then $F(\alpha)=\{i\}$.

Such a voting rule $\mathrm{F}: \mathrm{Z}_{+}^{\mathrm{V}} \rightarrow \mathrm{K}$ can be represented as a scoring rule iff there exist functions $S_{i}: V \rightarrow R$ for every alternative $i$ in $K$, such that, for each distribution $\alpha$ in $Z_{+}^{V}$,

$$
\begin{aligned}
F(\alpha) & =\operatorname{argmax}_{j \in K} \sum_{v \in V} S_{j}(v) \alpha(v) \\
& =\left\{i \in K \mid \sum_{v \in V} S_{i}(v) \alpha(v)=\max _{j \in K} \sum_{v \in V} S_{j}(v) \alpha(v)\right\} .
\end{aligned}
$$

In the next section, we list three axiomatic properties, taken from Smith [1973] and Young [1975], which are sufficient to imply that a voting rule $\mathrm{F}: \mathrm{Z}_{+}^{\mathrm{V}} \rightarrow \mathrm{K}$ can be represented as a scoring rule. 
2. The basic axioms

The most important axiom in the derivations of Smith [1973] and Young [1975] has been called reinforcement by Moulin [1988]. To formulate this axiom, suppose that our voting rule is applied separately in two districts, and $\alpha$ is the distribution of votes in one district, while $\beta$ is the distribution of votes in the other district. If the two districts were merged into a larger union, then $\alpha+\beta$ would be the resulting distribution of votes in the union. (We add vote distributions as vectors; that is, for any two vote distributions $\alpha$ and $\beta$, and for any real number $\mathrm{n}, \alpha+\beta$ and $\mathrm{n} \alpha$ are the vote distributions such that $(\alpha+\beta)(v)=\alpha(v)+\beta(v)$, and $(n \alpha)(v)=n \times \alpha(v)$.) Suppose now that some alternative $i$ would win in both districts separately. We may expect that this alternative $i$ should also win in the union of the two districts; that is, $F(\alpha) \cap F(\beta)$ should be a subset of $F(\alpha+\beta)$. Furthermore, we may expect that nothing that loses to this alternative $i$ in either district should be admitted as a tied winner in the union; that is, $F(\alpha+\beta)$ should be a subset of $F(\alpha) \cap$ $F(\beta)$, when this intersection is nonempty.

Axiom 1 (Reinforcement). For any vote distributions $\alpha$ and $\beta$ in $Z_{+}^{V}$, if $F(\alpha) \cap F(\beta) \neq \varnothing$ then $F(\alpha+\beta)=F(\alpha) \cap F(\beta)$.

Our second axiom has been called an Archimedean or continuity condition by Smith [1973] and Young [1975], but we refer to it here as an overwhelming-majority axiom, to emphasize its motivation. Given any two distributions $\alpha$ and $\beta$ in $z_{+}^{V}$, let $n$ be a very large positive integer, and consider the distribution n $\alpha+\beta$. This distribution represents the result when the set of voters can be partitioned into $n+1$ blocs, $n$ of which are submitting the vote distribution $\alpha$, and one of which is submitting the 
distribution $\beta$. By reinforcement, if the $\beta$ bloc were removed from the electorate, the outcome would be $F(n \alpha)=F(\alpha)$. So $n \alpha$ is the union of many blocs of voters which are uniformly endorsing the alternatives in $F(\alpha)$. When $n$ becomes very large, the $\beta$ bloc becomes an infinitesimal portion of the electorate, and such an infinitesimal bloc should not be able to overturn the decision of the overwhelming majority n $\alpha$. We may suppose that the only possible effect of such an infinitesimal minority should be to make some selection among the tied winners, if the overwhelming majority has generated a tie among two or more alternatives.

Axiom 2 (Overwhelming majority). For any distributions $\alpha$ and $\beta$ in $z_{+}^{V}$, there exists a positive integer $\mathrm{N}$ such that, for every integer $\mathrm{n}$ that is greater than $\mathrm{N}$,

$$
\mathrm{F}(\mathrm{n} \alpha+\beta) \subseteq \mathrm{F}(\alpha) .
$$

The third assumption is that the voting rule treats the various alternatives symmetrically. For any way of relabeling the alternatives, there should be a way of relabeling the possible votes such that the voting rule looks the same. A relabeling of the alternatives is a permutation of $\mathrm{K}$, that is, a one-to-one mapping $\pi: K \rightarrow K$ of the set of alternatives onto itself. A relabelling of the votes is a permutation of the set $V$, that is, a one-to-one mapping $\pi^{*}: \mathrm{V} \rightarrow \mathrm{V}$ of the set of possible votes onto itself. Given any permutation of the votes $\pi^{*}: \mathrm{V} \rightarrow \mathrm{V}$, for any distribution $\alpha$ in $\mathrm{Z}_{+}^{\mathrm{V}}$ we may let $\pi^{*}(\alpha)$ denote the distribution such that

$$
\left(\pi^{*}(\alpha)\right)\left(\pi^{*}(\mathrm{v})\right)=\alpha(\mathrm{v}), \quad \forall \mathrm{v} \in \mathrm{V}
$$

That is, letting [v] denote the distribution that consists of only one $v$ vote and no other votes, we can write 


$$
\alpha=\Sigma_{\mathrm{V} \in \mathrm{V}} \alpha(\mathrm{v})[\mathrm{v}] \text { and } \pi^{*}(\alpha)=\Sigma_{\mathrm{v} \in \mathrm{V}} \alpha(\mathrm{v})\left[\pi^{*}(\mathrm{v})\right]
$$

Axiom 3 (Neutrality). For any permutation of the alternatives $\pi: K \rightarrow K$, there exists a permutation of the votes $\pi^{*}: \mathrm{V} \rightarrow \mathrm{V}$ such that, for every distribution $\alpha$ in $\mathrm{Z}_{+}^{\mathrm{V}}$,

$$
\mathrm{F}\left(\pi^{*}(\alpha)\right)=\{\pi(i) \mid i \in F(\alpha)\}
$$

When we assume this neutrality axiom, we can also assume without loss of generality that the derivation of $\pi^{\dot{*}}$ from $\pi$ preserves the composition of functions. That is, if $\pi_{1}$ and $\pi_{2}$ are any two permutations of the alternatives and if $\pi_{1}{ }^{*}$ and $\pi_{2}{ }^{*}$ are the corresponding permutations of the votes that satisfy the neutrality condition, then we can satisfy the neutrality condition for the permutation $\pi_{1} \circ \pi_{2}$ by letting

$$
\left(\pi_{1} \circ \pi_{2}\right)^{*}=\pi_{1}^{*} \circ \pi_{2}^{*} \text {, }
$$

because, for any distribution $\alpha$,

$$
\begin{aligned}
& \left\{\left(\pi_{1} \circ \pi_{2}\right)(i) \mid i \in F(\alpha)\right\}=\left\{\pi_{1}\left(\pi_{2}(i)\right) \mid i \in F(\alpha)\right\} \\
& \quad=\left\{\pi_{1}(j) \mid j \in F\left(\pi_{2}{ }^{*}(\alpha)\right)\right\}=F\left(\pi_{1}{ }^{*}\left(\pi_{2}{ }^{*}(\alpha)\right)=F\left(\left(\pi_{1}{ }^{*} \circ \pi_{2}{ }^{*}\right)(\alpha)\right) .\right.
\end{aligned}
$$

It is straightforward to verify that, if $F$ can be represented as a scoring rule then $F$ must satisfy the reinforcement and overwhelming-majorities axioms. The main result of this paper is that these axioms with neutrality are also sufficient to guarantee that $F$ can be represented as a scoring rule.

Theorem. If a voting rule $\mathrm{F}: \mathrm{Z}_{+}^{\mathrm{V}} \rightarrow \mathrm{K}$ satisfies Axioms 1,2 , and 3 , then it can be represented as a scoring rule.

The proof of this theorem is deferred to section 5. 


\section{Other axioms}

The reinforcement axiom can be derived from other considerations that involve the strategic implications of the voting rule for voters' decision-making. Consider a bloc of voters who are planning their votes together, and are comparing two plans for how to vote. Let $\alpha$ denote the distribution of votes that the bloc would cast under the first plan, and let $\beta$ denote the distribution of votes that the bloc would cast under the second plan. (A plan might involve some abstentions, so $\sum_{\mathrm{v} \in \mathrm{V}} \alpha(\mathrm{v})$ does not have to equal $\left.\Sigma_{\mathrm{v} \in \mathrm{V}} \beta(\mathrm{v}).\right)$ The outcome of the election under either plan will also depend on the distribution of votes that are cast by the other individuals outside of the bloc. We may say that the change from $\alpha$ to $\beta$ can support alternative $j$ against alternative $i$ iff there exists some distribution $\gamma$ such that

$$
i \in F(\gamma+\alpha) \text { and } j \in F(\gamma+\beta) \text {. }
$$

That is, the proposed change from $\alpha$ to $\beta$ can support $j$ against $i$ if, with some fixed distribution of votes from the others in the electorate, $i$ would be in the choice set when the bloc's vote is $\alpha$, but $j$ would be in the choice set when the bloc's vote is $\beta$.

A stronger version of this definition should exclude the neutral case where both alternatives $i$ and $j$ are in both choice sets $F(\gamma+\alpha)$ and $F(\gamma+\beta)$. So we may say that the change from $\alpha$ to $\beta$ can strongly support $j$ against if if there exists some distribution $\gamma$ such that

$$
i \in F(\gamma+\alpha), \quad j \in F(\gamma+\beta), \quad \text { and }\{i, j\} \not F(\gamma+\alpha) \cap F(\gamma+\beta) \text {. }
$$

Decision-making by a bloc of voters is complicated by the fact that, when they are uncertain about others' votes, a proposed change in the bloc's vote could have many possible effects. To keep such complications within some 
bound, however, we might at least ask that, if a change from $\alpha$ to $\beta$ can strongly support alternative $j$ against alternative $i$ in one context, then there should not exist some other context in which the same change could support $i$ against $j$. That is, we may pose the following axiom.

Axiom 1a (Nonreversa1). For any distributions $\alpha, \beta, \gamma_{1}$, and $\gamma_{2}$ in $z_{+}^{V}$, and for any alternatives $i$ and $j$ in $k$, if

$$
\text { i } \in F\left(\gamma_{1}+\alpha\right), \quad j \in F\left(\gamma_{1}+\beta\right), \quad j \in F\left(\gamma_{2}+\alpha\right), \quad \text { and } i \in F\left(\gamma_{2}+\beta\right) \text {, }
$$

then $\{i, j\} \subseteq F\left(\gamma_{1}+\alpha\right) \cap F\left(\gamma_{1}+\beta\right)$, and $\{i, j\} \subseteq F\left(\gamma_{2}+\alpha\right) \cap F\left(\gamma_{2}+\beta\right)$.

This nonreversal axiom must be satisfied by all scoring rules, because a change from $\alpha$ to $\beta$ can strongly support $j$ against $i$ in a scoring rule only if the net difference between the total scores of $j$ and $i$ is greater in $\beta$ than in $\alpha$. Indeed, it can be shown that the reinforcement axiom by itself implies the nonreversal axiom. With one minor technical condition, we can also show that the nonreversal axiom implies the reinforcement axiom.

Let $\overrightarrow{0}$ denote the zero vector in $\mathcal{R}^{\mathrm{V}}$, which is the vote distribution when nobody votes. Notice that the neutrality axiom and the overwhelming-majority axiom both imply that $\mathrm{F}(\overrightarrow{0})=\mathrm{K}$.

Proposition 1. If $\mathrm{F}: \mathrm{Z}_{+}^{\mathrm{V}} \rightarrow \mathrm{K}$ satisfies Axiom $\mathrm{la}$, and $\mathrm{F}(\overrightarrow{\mathrm{O}})=\mathrm{K}$, then $\mathrm{F}$ satisfies Axiom 1.

Proof. Suppose $F(\alpha) \cap F(\beta) \neq \varnothing$, and let i be any alternative such that $i \in F(\alpha) \cap F(\beta)$. Let $j$ be any alternative such that $j \in F(\alpha+\beta)$. Now consider the facts that $i \in F(\beta), j \in F(\alpha+\beta), j \in F(\overrightarrow{0})$, and $i \in F(\alpha)$. Then applying Axiom $1 \mathrm{a}$ (with $\gamma_{1}=\beta$ and $\gamma_{2}=\overrightarrow{0}$, and with $\overrightarrow{0}$ and $\alpha$ here taking the roles of $\alpha$ and $\beta$ in Axiom la respectively), we get 


$$
\{i, j\} \subseteq F(\beta) \cap F(\alpha+\beta), \text { and }\{i, j\} \subseteq F(\overrightarrow{0}) \cap F(\alpha)
$$

So $j \in F(\alpha) \cap F(\beta)$, and $i \in F(\alpha+\beta)$. But we originally selected $j$ in $F(\alpha+\beta)$ and $i$ in $F(\alpha) \cap F(\beta)$. So $F(\alpha+\beta)=F(\alpha) \cap F(\beta) . \quad$ Q.E.D.

In our derivation of scoring rules, the neutrality axiom operates only through two conditions on the range of $F$, which are worth formalizing explicitly. We label these conditions as Axioms $3 a$ and $3 b$, to indicate their close relationship with the neutrality axiom.

Axiom $3 a$ (Weak range condition). For every alternative $i$ in $K$, there is a distribution $\mu_{i}$ in $z_{+}^{V}$ such that $F\left(\mu_{i}\right)=\{i\}$.

Axiom $3 \mathrm{~b}$ (Strong range condition). For every set $\mathrm{L}$ that is a nonempty subset of $\mathrm{K}$, there exists some distribution $\gamma_{L}$ in $Z_{+}^{V}$ such that $F\left(\gamma_{L}\right)=L$ and $\gamma_{L}(v)>0, \quad \forall v \in V$

A voting rule that always selects $F(\alpha)=\mathrm{K}$, for every distribution $\alpha$ in $\mathrm{Z}_{+}^{\mathrm{V}}$, would violate both of these conditions. Once this trivial rule is excluded, however, we find that Axiom $3 a$ and Axiom $3 b$ follow from Axioms 1, 2, and 3 .

Proposition 2. Let $\mathrm{F}: \mathrm{Z}_{+}^{\mathrm{V}} \rightarrow \rightarrow \mathrm{K}$ be a voting rule that does not always select the set of all alternatives $K$. (That is, $F(\alpha) \neq K$ for some $\alpha$ in $z_{+}^{V}$ ) If $F$ satisfies Axioms 1, 2, and 3, then it satisfies Axioms $3 a$ and $3 b$.

Proof. We first prove Axiom 3a. Let L denote a set of minimal size such that $F(\alpha)=$ L for at least one distribution $\alpha$. L is nonempty, because our definition of a voting rules specifies that it always selects a nonempty winning set. By the nontriviality assumption, $L \neq K$. If $\# L=1$, then we are 
done. So suppose that $\# \geq 2$. Let $i$ and $j$ be two distinct alternatives such that $i \in L$ and $j \in L$, and let $h$ be an alternative such that $h \notin L$. Let $\pi_{h i}$ denote the permutation of the alternatives that switches $h$ and $i$, leaving all other alternatives fixed. By the neutrality axiom, $\mathrm{F}\left(\pi_{\mathrm{hi}}{ }^{*}(\alpha)\right)=\mathrm{L} \cup\{\mathrm{h}\} \backslash\{\mathrm{i}\}$. Then by the reinforcement axiom,

$$
\mathrm{L} \backslash\{\mathrm{i}\}=\mathrm{F}(\alpha) \cap \mathrm{F}\left(\pi_{\mathrm{hi}}{ }^{*}(\alpha)\right)=\mathrm{F}\left(\alpha+\pi_{\mathrm{hi}}{ }^{*}(\alpha)\right) \text {. }
$$

because this intersection contains $j$ and so is nonempty. But $L \backslash\{i\}$ is a set with fewer members than $\mathrm{L}$, which contradicts the minimality of $\mathrm{L}$. This contradiction proves Axiom 3a.

We now prove Axiom 3b. As above, for any two distinct alternatives $i$ and $j$, let $\pi_{i j}$ denote the permutation of $k$ that switches $i$ and $j$ but keeps all other alternatives fixed. For any alternative $i$, let $P(i)$ denote the set of all permutations of the alternatives $\pi: K \rightarrow K$ that keep $i$ fixed, in the sense that $\pi(i)=i$.

Let $\mathrm{g}$ denote a fixed alternative in $\mathrm{K}$. By Axiom $3 \mathrm{a}$, we can select a distribution $\mu_{\mathrm{g}}$ such that $F\left(\mu_{\mathrm{g}}\right)=\{\mathrm{g}\}$. By neutrality, $F\left(\pi^{*}\left(\mu_{\mathrm{g}}\right)\right)=\{\mathrm{g}\}$ for any $\pi$ in $\mathrm{P}(\mathrm{g})$. Let $\Omega$ be the distribution such that $\Omega(\mathrm{v})=1$ for all $\mathrm{v}$. By neutrality, we must have $F(\Omega)=\mathrm{K}$, because $\pi^{*}(\Omega)=\Omega$ for all permutations. Let

$$
\theta_{\mathrm{g}}=\Omega+\Sigma_{\pi \in \mathrm{P}(\mathrm{g})} \pi^{*}\left(\mu_{\mathrm{g}}\right) \text {. }
$$

Reinforcement then implies that $F\left(\theta_{g}\right)=\{g\}$, because $\theta_{g}$ is the sum of $(\# \mathrm{~K}-1)$ ! distributions in $\mathrm{g}$ is the unique winner, plus one distribution in which all alternatives win. The symmetry of the construction guarantees that

$$
\pi^{*}\left(\theta_{g}\right)=\theta_{g}, \quad \forall \pi \in P(g) .
$$

Furthermore, for every $\mathrm{v}$ in $\mathrm{V}, \theta_{\mathrm{g}}(\mathrm{v}) \geq \Omega(\mathrm{v})>0$.

For any other alternative $i$, let $\theta_{i}=\pi_{i g} \dot{*}\left(\theta_{g}\right)$. By neutrality, we have $F\left(\theta_{i}\right)=\{i\}$, because $\pi_{i g}$ exchanges $i$ and $g$. For any set of alternatives $L$, let 


$$
\gamma_{\mathrm{L}}=\Sigma_{i \in \mathrm{L}}{ }^{\theta}{ }_{\mathrm{i}}
$$

We now prove the claim that $F\left(\gamma_{L}\right)=L$, for all L.

If this claim fails to hold, then let $\mathrm{L}$ be a set of minimal size for which it fails. It is straightforward to check that, if $i$ and $j$ are both in $L$, or if $i$ and $j$ are both in $K \backslash L$, then $\pi_{i j}{ }^{*}\left(\gamma_{L}\right)=\gamma_{L}$. So the neutrality axiom implies that $F\left(\gamma_{L}\right)$ must equal either $L$ or $K \backslash L$ or $K$. Thus, if the claim fails then we can choose some alternative $h$ such that

$h \in F\left(\gamma_{L}\right)$ but $h \notin L$.

Let us also pick two distinct alternatives $i$ and $j$ that are both in $L$. (The claim cannot fail when $\#$ equals 1 , because $\left.F\left(\gamma_{\{i\}}\right)=F\left(\theta_{i}\right)=\{i\}.\right)$ By minimality of $\mathrm{L}$, we know that

$$
F\left(\gamma_{L \backslash\{i\}}\right)=L \backslash\{i\}
$$

and so

$h \notin F\left(\gamma_{L \backslash\{i\}}\right)$ and $j \in F\left(\gamma_{L \backslash\{i\}}\right)$

Now exchange $h$ and $j$ by the permutation $\pi_{h j}$. By the symmetry of the $\theta$ and $\gamma$ distributions, we have

$$
\begin{aligned}
& \pi_{h j}{ }^{*}\left(\gamma_{L \backslash\{i\}}\right)=\gamma_{L \cup\{h\} \backslash\{i, j\}} \\
& \pi_{h j}{ }^{*}\left(\gamma_{L}\right)=\gamma_{L \cup\{h\} \backslash\{j\}}=\gamma_{L \cup\{h\} \backslash\{i, j\}}+\theta_{i} .
\end{aligned}
$$

Thus,

$$
\begin{aligned}
& h=\pi_{h j}(j) \in F\left(\gamma_{L \cup\{h\} \backslash\{i, j\}}\right), \quad j=\pi_{h j}(h) \notin F\left(\gamma_{L \cup\{h\} \backslash\{i, j\}}\right), \\
& j=\pi_{h j}(h) \in F\left(\pi_{h j}{ }^{*}\left(\gamma_{L}\right)\right)=F\left(\gamma_{L \cup\{h\} \backslash\{j\}}\right) .
\end{aligned}
$$

Because $j$ is in both $F\left(\gamma_{L \backslash\{i\})}\right.$ and $F\left(\gamma_{L \cup\{h\} \backslash\{j\}}\right)$ and $h$ is not in $F(L \backslash\{i\})$, the reinforcement axiom implies that

$$
h \notin F\left(\gamma_{L \backslash\{i\}}+\gamma_{L U\{h \backslash \backslash\{j\}}\right)=F\left(\gamma_{L \backslash\{i\}}\right) \cap F\left(\gamma_{L \cup\{h\} \backslash\{j\}}\right) .
$$

On the other hand $h$ is in both $F\left(\gamma_{L}\right)$ and $F\left(\gamma_{L \cup\{h \backslash \backslash i, j\}}\right)$, and so

$$
h \in F\left(\gamma_{L}+\gamma_{L U\{h\} \backslash\{i, j\}}\right)=F\left(\gamma_{L}\right) \cap F\left(\gamma_{L \cup\{h\} \backslash\{i, j\}}\right) .
$$


However,

$$
\gamma_{L}-\gamma_{L \backslash\{i\}}=\theta_{i}=\gamma_{L \cup\{h\} \backslash\{j\}}-\gamma_{L \cup\{h\} \backslash\{i, j\}}
$$

and so

$$
\gamma_{L}+\gamma_{L \cup\{h\} \backslash\{i, j\}}=\gamma_{L \backslash\{i\}}+\gamma_{L \cup\{h\} \backslash\{j\}} .
$$

This contradiction implies that the claim cannot fail for any set L. That is, $F\left(\gamma_{L}\right)=L$, for all L, and so Axiom $3 b$ is satisfied.

Q.E.D.

\section{Implications of Axioms 1,2 , and $3 a$}

Nonneutral voting rules which satisfy the reinforcement axiom, the overwhelming majority axiom, and the weak range condition are not necessarily scoring rules, but they have a related representation. Instead of having one scoring function $\mathrm{S}_{i}$ for each alternative $i$, we have a bilateral balance function $s_{i j}$ for each pair of alternatives $(i, j)$. Then an alternative $j$ wins iff its total balance $\Sigma_{v \in V} s_{i j}(v) \alpha(v)$ is nonnegative against every other alternative i. Any scoring rule can also be represented in this bilateral balance form, by letting $s_{i j}(v)=s_{j}(v)-s_{i}(v)$, but the bilateral balance form is strictly more general. (See Example 2 in Section 6.)

Proposition 3. Suppose that $\mathrm{F}: \mathrm{Z}_{+}^{\mathrm{V}} \rightarrow \mathrm{K}$ is a voting rule which satisfies Axioms 1, 2, and 3a. Then there exist functions there exist functions $s_{i j}: V \rightarrow R$, for every pair of distinct alternatives $i$ and $j$ in $K$, such that, for each distribution $\alpha$ in $z_{+}^{V}$

$$
F(\alpha)=\left\{j \mid \sum_{v \in V} s_{i j}(v) \alpha(v) \geq 0, \forall i \in K \backslash\{j\}\right) .
$$

Furthermore, for each $i$ and $j$,

$$
s_{j i}(v)=-s_{i j}(v), \quad \forall v \in v,
$$

and there exists some $w$ in $V$ such that $s_{i j}(w) \neq 0$. 
Proof. Consider a pair of distinct alternatives $i$ and $j$. Let

$$
\mathrm{D}_{i j}=\left\{\beta-\alpha \mid \alpha \in \mathrm{Z}_{+}^{\mathrm{V}}, \beta \in \mathrm{Z}_{+}^{\mathrm{V}}, \mathrm{F}(\beta)=\{\mathrm{j}\} \text {, and } \mathrm{F}(\alpha)=\{i\}\right\} \text {. }
$$

$D_{i j}$ is a subset of $R^{V}$. Let $C_{i j}$ denote the convex hull of the set $D_{i j}$.

In the vector space $\boldsymbol{R}^{\mathrm{V}}$, the vector $\overrightarrow{0}$ cannot be in the convex set $C_{i j}$. If it were, then there would exist distributions $\left(\alpha_{1}, \ldots, \alpha_{M}, \beta_{1}, \ldots, \beta_{M}\right)$, each of which is in $Z_{+}^{V}$, such that

$$
F\left(\alpha_{m}\right)=\{i\} \text { and } F\left(\beta_{m}\right)=\{j\}, \quad \forall m,
$$

and such that there is a nonnegative solution $\left(\lambda_{1}, \ldots, \lambda_{M}\right)$ to the equations

$$
\sum_{\mathrm{m}=1}^{\mathrm{M}} \lambda_{\mathrm{m}}\left(\alpha_{\mathrm{m}}(\mathrm{v})-\beta_{\mathrm{m}}(\mathrm{v})\right)=0, \quad \forall \mathrm{v} \in \mathrm{V}
$$

However, this system of equations is linear and homogeneous, and it has all

integer coefficients (because $\alpha_{\mathrm{m}}(\mathrm{v})$ and $\beta_{\mathrm{m}}(\mathrm{v})$ are integers), so having

nonnegative solutions in the real numbers implies that it also has nonnegative solutions in the rational numbers and in the integers. So there exist

nonnegative integers $\left(\lambda_{1}, \ldots, \lambda_{M}\right)$ such that

$$
\Sigma_{\mathrm{m}=1}^{\mathrm{M}} \lambda_{\mathrm{m}} \alpha_{\mathrm{m}}=\Sigma_{\mathrm{m}=1}^{\mathrm{M}} \lambda_{\mathrm{m}} \beta_{\mathrm{m}} .
$$

But the reinforcement axiom implies that

$$
F\left(\Sigma_{\mathrm{m}=1}^{\mathrm{M}} \lambda_{\mathrm{m} m} \alpha_{\mathrm{m}}\right)=\{i\} \neq\{j\}=F\left(\sum_{\mathrm{m}=1}^{\mathrm{M}} \lambda_{\mathrm{m}} \beta_{\mathrm{m}}\right) .
$$

This contradiction implies that $\overrightarrow{0} \notin C_{i j}$.

Thus, by the Supporting Hyperplane Theorem, we can choose a vector $s_{i j}=\left(s_{i j}(v)\right)_{v \in V}$ in $k^{V}$ such that

$$
s_{i j} \neq \overrightarrow{0}
$$

and, for all $\alpha$ and $\beta$ in $Z_{+}^{V}$ such that $F(\beta)=\{j\}$ and $F(\alpha)=\{i\}$,

$$
\sum_{v \in V} s_{i j}(v)(\beta(v)-\alpha(v)) \geq 0
$$

To satisfy these conditions when the roles of $i$ and $j$ are reversedWe can simply let $s_{j i}=-s_{i j}$.

It now remains only for us to show that these vectors $s_{i j}$ characterize $F$ 
as asserted in Proposition 3. We do so by proving a series of four claims.

Claim 1. If $F(\beta)=\{j\}$ then $\Sigma_{v \in V} s_{i j}(v) \beta(v) \geq 0, \quad \forall i \in K \backslash\{j\}$.

To prove this claim, use the weak range condition to pick $\mu_{i}$ such that

$F\left(\mu_{i}\right)=\{i\}$. By the overwhelming-majority axiom (and nonemptiness of $F$ ), there exist some positive integer $n$ such that $F\left(n \beta+\mu_{i}\right)=\{j\}$. So

$$
\Sigma_{v \in V} s_{i j}(v)(n \beta(v)+\alpha(v)-\alpha(v)) \geq 0,
$$

which implies the inequalities described in Claim 1.

Claim 2. If $j \in F(\beta)$ then $\sum_{v \in V} s_{i j}(v) \beta(v) \geq 0, \quad \forall i \in K \backslash\{j\}$.

Suppose that $j \in F(\beta)$. Pick $\mu_{j}$ so that $F\left(\mu_{j}\right)=\{j\}$. Using the reinforcement axiom, we can inductively show that, for any positive integer $\mathrm{n}$,

$$
F\left(n \beta+\mu_{j}\right)=\{j\} .
$$

So we must have

$$
\sum_{v \in V} s_{i j}(v)\left(n \beta(v)+\mu_{j}(v)\right) \geq 0, \quad \forall i \in K \backslash\{j) .
$$

This inequality cannot hold for all arbitrarily large $n$ unless

$$
\Sigma_{v \in V} s_{i j}(v) \beta(v) \geq 0, \quad \forall i \in K \backslash\{j\},
$$

as the claim requires.

Claim 3. For any alternative $j$, there exists some $\bar{\mu}_{j}$ such that $F\left(\mu_{j}\right)=\{j\}$ and

$$
\Sigma_{v \in V} s_{i j}(v) \bar{\mu}_{j}(v)>0, \quad \forall i \in K \backslash\{j\}
$$

To prove this claim, use the weak range condition to pick $\mu_{j}$ such that $F\left(\mu_{j}\right)=\{j\}$. Because $s_{i j} \neq \overrightarrow{0}$, we can pick an alternative w such that $s_{i j}(w) \neq 0$, and let $[w]$ denote the vote distribution that consists of a single $w$ vote and no other votes. By the overwhelming-majority axiom, we can find a positive number $\mathrm{n}$ such that

$$
\{j\}=F\left(n \mu_{j}\right)=F\left(n \mu_{j}+[w]\right)=F\left(n \mu_{j}+2[w]\right) .
$$

So by Claim 1 , 


$$
\sum_{v \in V} s_{i j}(v) n \mu_{j}(v)+m s_{i j}(w) \geq 0, \text { for } m \in\{0,1,2\} .
$$

But $s_{i j}(w) \neq 0$, and so we must have

$$
\Sigma_{v \in V} s_{i j}(v) n \mu_{j}(v)+s_{i j}(w)>0 .
$$

So let $\bar{\mu}_{j}=n \mu_{j}+[\mathrm{w}]$, and Claim 3 is proven.

Claim 4. For any $j$ in $K$ and any $\beta$ in $z_{+}^{V}$, if

$$
\sum_{v \in V} s_{i j}(v) \beta(v) \geq 0, \quad \forall i \in K \backslash\{j\},
$$

then $j \in F(\beta)$.

Suppose that $\beta$ satisfies the inequalities in the hypothesis of the claim.

Pick $\bar{\mu}_{j}$ satisfying the conditions of Claim 3. For any positive integer $\mathrm{m}$,

$$
\Sigma_{v \in V} s_{i j}(v)\left(m \beta(v)+\bar{\mu}_{j}(v)\right)>0, \quad \forall i \in K \backslash\{j\} .
$$

Using the fact that $s_{j i}=-s_{i j}$, we have

$$
\Sigma_{v \in V} s_{j i}(v)\left(m \beta(v)+\bar{\mu}_{j}(v)\right)<0, \quad \forall i \in K \backslash(j),
$$

and so, by Claim 2,

$$
i \notin F\left(m \beta+\bar{\mu}_{j}\right), \quad \forall i \in K \backslash\{j\} .
$$

By the nonemptiness of $F$, we must have $F\left(m \beta+\bar{\mu}_{j}\right)=\{j\}$. But the overwhelming-majority axiom implies that $F\left(m \beta+\bar{\mu}_{j}\right) \subseteq F(\beta)$ for al1 sufficiently large $\mathrm{m}$. Thus $\mathrm{j} \in \mathrm{F}(\beta)$, and so the claim is proven.

Claims 2 and 4 together assert that $j \in F(\beta)$ if and only if

$$
\Sigma_{v \in V} s_{i j}(v) \beta(v) \geq 0, \quad \forall i \in K \backslash\{j\},
$$

and so the proof of Proposition 3 is complete.

Q.E.D.

\section{Derivation of scoring rules}

In this section, we show that Axioms 1, 2, and $3 b$ are sufficient to imply

that $\mathrm{F}: \mathrm{Z}_{+}^{\mathrm{V}} \rightarrow \mathrm{K}$ is a scoring rule. The main theorem will then follow immediately from Propositions 2 and 4. 
Proposition 4. If $\mathrm{F}: \mathrm{Z}_{+}^{\mathrm{V}} \rightarrow \mathrm{K}$ satisfies Axioms 1,2 , and $3 \mathrm{~b}$, then it can be represented as a scoring rule.

Proof. Let F satisfy Axioms 1, 2, and 3b. Axiom 3b obviously implies Axiom 3a, so we can let $s_{i j}$ be as in Proposition 3, for each pair of distinct alternatives $i$ and $j$. Using the stronger range condition, we now prove two more claims.

Claim 5. If $j \in F(\beta)$ and $i \notin F(\beta)$ then

$$
\sum_{v \in V} s_{i j}(v) \beta(v)>0 \text {. }
$$

To prove the claim, let $\gamma_{\{i, j\}}$ be as in Axiom $3 b$. That is,

$$
F\left(\gamma_{\{i, j\}}\right)=\{i, j\}, \text { and } \gamma_{\{i, j\}}(v)>0, \quad \forall v \in V
$$

By Proposition 3 (using the fact that $s_{j i}=-s_{i j}$ ), we must have

$$
\Sigma_{v \in V} s_{i j}(v) \gamma_{\{i, j\}}(v)=0
$$

By reinforcement, we have

$$
F\left(\gamma_{\{i, j\}}+\beta\right)=\{j\} .
$$

Pick any $w$ such that $s_{i j}(w) \neq 0$. Let $\varepsilon(w)=-1$ if $s_{i j}(w)>0$, let $\varepsilon(w)=1$ if $s_{i j}(w)<0$, and let $\varepsilon(v)=0$ for every $v \neq w$. Because $\gamma_{\{i, j\}}(w) \geq 1, \gamma_{\{i, j\}}+\varepsilon$ is a distribution in $z_{+}^{V}$. By the overwhelming-majority axiom, there exists some positive integer $\mathrm{n}$ such that

$$
F\left(n\left(\gamma_{\{i, j\}}+\beta\right)+\left(\gamma_{\{i, j\}}+\varepsilon\right)\right)=\{j\} .
$$

So by Proposition 3 ,

$$
\begin{gathered}
0 \leq \Sigma_{v \in V} s_{i j}(v)\left(n\left(\gamma_{\{i, j\}(v)}+\beta(v)\right)+\left(\gamma_{\{i, j\}(v)}+\varepsilon(v)\right)\right) \\
=n \Sigma_{v \in V} s_{i j}(v) \beta(v)-\left|s_{i j}(w)\right| .
\end{gathered}
$$

The strict inequality in the claim immediately follows, proving claim 5.

Claim 6. For any three distinct alternatives $h, i$, and $j$, the system of inequalities inequalities 


$$
\begin{aligned}
& \sum_{v \in V} s_{h i}(v) \delta(v)>0 \\
& \sum_{v \in V} s_{i j}(v) \delta(v)>0 \\
& \sum_{v \in V} s_{j h}(v) \delta(v)>0
\end{aligned}
$$

cannot have any solution $\delta$ in $R^{\mathrm{V}}$.

If this system of inequalities had any feasible solutions $\delta$ in the real numbers, then it would also have solutions in the rational numbers (by continuity of linear functions), and so (by homogeneity) it would also have a solution $\delta$ such that $\delta(\mathrm{v})$ is an integer for all $\mathrm{v}$. But then considex the distribution $\gamma_{\{h, i, j\}}$ from Axion 3b. Let

$$
\mathrm{m}=\max _{\mathrm{v} \in \mathrm{V}}|\delta(\mathrm{v})| \text {, }
$$

and observe that

$$
\mathrm{m}_{\{h, i, j\}}+\delta \in \mathrm{Z}_{+}^{\mathrm{V}} .
$$

So by the overwhelming-majority axiom, there exists some positive integer $n$ such that

$$
F\left(n \gamma_{\{h, i, j)}+m \gamma_{\{h, i, j\}}+\delta\right) \subseteq F\left(\gamma_{\{h, i, j\}}\right)=\{h, i, j\} .
$$

By Proposition 3, we must have

$$
\Sigma_{v \in V} s_{h i}(v) \gamma_{\{h, i, j\}}(v)=0,
$$

because $h \in F\left(\gamma_{\{h, i, j\}}\right), i \in F\left(\gamma_{\{h, i, j\}}\right)$, and $s_{i h}=-s_{h i}$. Thus, the first inequality in the claim implies that

$$
\Sigma_{v \in V} s_{h i}(v)\left((n+m) \gamma_{\{h, i, j\}}(v)+\delta(v)\right)>0,
$$

and so Proposition 3 implies that

$$
\mathrm{h} \notin \mathrm{F}\left(\mathrm{n} \gamma_{\{\mathrm{h}, \mathrm{i}, \mathrm{j}\}}+\mathrm{m}_{\{\mathrm{h}, \mathrm{i}, \mathrm{j}\}}+\delta\right) .
$$

A similar argument using the second inequality (and permuting the roles of $h$, i, and j) implies that

$$
i \notin F\left(n \gamma\{h, i, j\}+m \gamma_{\{h, i, j\}}+\delta\right),
$$

Similarly, the third inequality similarly implies that 


$$
j \notin F\left(n \gamma\{h, i, j\}+m \gamma_{\{h, i, j\}}+\delta\right) .
$$

But these conclusions contradict the assumption that a voting rule cannot have an empty choice set. Thus, the system of inequalities cannot have any solution $\delta$. So we have proven Claim 6 .

To complete the proof of Proposition 4, now let $h$ be any fixed alternative in $\mathrm{K}$. From the strong range condition (Axiom 3b), consider the distribution $\gamma_{K \backslash\{h\}}$, for which every alternative other than $h$ wins. For each $j$ in $K \backslash\{h\}$, let

$$
\Gamma_{j}=\Sigma_{v \in V} s_{h j}(v) \gamma_{K \backslash\{h)}(v)
$$

Claim 5 then implies that

$$
\Gamma_{j}>0, \quad \forall j \in K \backslash\{h\} .
$$

By Proposition 3, we must also have

$$
\Sigma_{v \in V} s_{i j}(v) \gamma_{K \backslash\{h\}}(v)=0, \quad \forall i \in K \backslash\{h, j\}, \quad \forall j \in K \backslash\{h\} .
$$

Now fix any two distinct alternatives $i$ and $j$ such that $i \neq h \neq j$. Claim 6 implies that the following linear programming problem has no feasible solutions:

$$
\begin{aligned}
& \operatorname{minimize} 0 \\
& \text { subject to } \delta \in \boldsymbol{R}^{V}, \\
& \sum_{v \in V} s_{h i}(v) \delta(v) \geq 1, \\
& \sum_{v \in V} s_{i j}(v) \delta(v) \geq 1, \\
& \sum_{v \in V}-s_{h j}(v) \delta(v) \geq 1 .
\end{aligned}
$$

The dual of this linear programming problem can be written as follows:

$$
\begin{aligned}
& \operatorname{maximize} x_{i j}+y_{i j}+z_{i j} \\
& \text { subject to } x_{i j} \geq 0, \quad y_{i j} \geq 0, \quad z_{i j} \geq 0, \\
& x_{i j} s_{h i}(v)+y_{i j} s_{i j}(v)-z_{i j} s_{h j}(v)=0, \quad \forall v \in v .
\end{aligned}
$$

By the Duality Theorem of linear programing, this dual problem cannot have a 
finite optimal solution, because the original problem is infeasible. But the dual problem does have a feasible solution (simply let $x_{i j}, y_{i j}$, and $z_{i j}$ all be zero). So the dual problem must have an unbounded optimum. Thus, we can pick nonnegative numbers $x_{i j}, y_{i j}$, and $z_{i j}$ such that the dual constraints are satisfied and

$$
x_{i j}+y_{i j}+z_{i j}>0 \text {. }
$$

These dual constraints imply that

$$
\begin{aligned}
0= & \sum_{v \in V}\left(x_{i j} s_{h i}(v)+y_{i j} s_{i j}(v)-z_{i j} s_{h j}(v)\right) \gamma_{K \backslash\{h\}}(v) \\
& =x_{i j} \Gamma_{i}+0-z_{i j} \Gamma_{j} .
\end{aligned}
$$

So $z_{i j}=\left(\Gamma_{i} / \Gamma_{j}\right) x_{i j}$. The number $x_{i j}$ cannot be zero, or else the dual constraints would then force all $\mathrm{s}_{i j}(v)$ to be zero, which would contradict Claim 5 (for any distribution where $j$ wins and $i$ does not). If $y_{i j}$ were equal to zero, then $s_{h i}(v)$ would equal $\left(\Gamma_{i} / \Gamma_{j}\right) s_{h j}(v)$ for all $v$, which is not possible, because it would contradict the fact that (again using Axiom $3 b$ and Claim 5)

$$
\begin{aligned}
& \sum_{v \in V} s_{h i}(v) \gamma_{\{h, j\}}(v)<0 \text { and } \sum_{v \in V} s_{h j}(v) \gamma_{\{h, j\}}(v)=0 . \\
& \text { So } x_{i j}>0 \text { and } y_{i j}>0 \text {. Let } \\
& r_{i j}=\Gamma_{i} x_{i j} / y_{i j} .
\end{aligned}
$$

Then the dual constraints can be rewritten

$$
s_{i j}(v)=r_{i j}\left(s_{h j}(v) / \Gamma_{j}-s_{h i}(v) / \Gamma_{i}\right), \quad \forall v \in v
$$

Now let

$$
\begin{aligned}
& \mathrm{s}_{i}(\mathrm{v})=\mathrm{s}_{\mathrm{hi}}(\mathrm{v}) / \Gamma_{i}, \quad \forall \mathrm{i} \in \mathrm{K} \backslash\{\mathrm{h}\}, \quad \forall \mathrm{v} \in \mathrm{v}, \\
& \mathrm{S}_{\mathrm{h}}(\mathrm{v})=0, \quad \forall \mathrm{v} \in \mathrm{v} .
\end{aligned}
$$

Because each $r_{i j}$ is a strictly positive number, Proposition 3 implies that $j \in F(\alpha)$ if and only if

$$
\sum_{v \in V}\left(S_{j}(v)-S_{i}(v)\right) \alpha(v) \geq 0, \quad \forall i \in K \backslash\{j\} .
$$


Thus, $\mathrm{F}: \mathrm{Z}_{+}^{\mathrm{V}} \rightarrow \mathrm{K}$ can be represented as a scoring rule.

Q.E.D.

Proof of the Theorem. If $F(\alpha)$ equals $\mathrm{K}$ for every vote distribution $\alpha$, then $\mathrm{F}$ can be trivially represented as a scoring rule, by letting the scoring functions be identically zero. So we may suppose that $F(\alpha)$ is not always $K$, in which case Axioms 1, 2, and 3 imply that Axiom $3 b$ is also satisfied. Then Proposition 4 asserts that $F$ can be represented as a scoring rule. Q.E.D.

\section{Examples}

Example 1. For an example of a voting rule which satisfies Axioms 1 and 3 but not Axiom 2 (overwhelming majorities), consider a voting system in which each voter's ballot must contain an ordered list of two alternatives. To win, an alternative must be listed in the first position on the maximal number of ballots; but if two or more alternatives are tied for being listed first on the most ballots, then the winners are those among these tied alternatives that are listed in the second position on the most ballots.

Under this voting rule, suppose that the distribution $\alpha$ generates a tie between alternatives $i$ and $j$ for being listed most often in the first position, but $F(\alpha)=\{i\}$ because $i$ is listed in the second position more than $j$. Let $\beta$ be a distribution containing one vote that lists $j$ in the first position and no other votes. Then for any positive integer $n, F(n \alpha+\beta)=\{j\}$, thus violating Axiom 2. This voting rule is not a scoring rule in the simple sense used in this paper, but it could be considered as generalized sequential scoring rule. Such generalized sequential scoring rules were also axiomatically derived by Smith [1973] and Young [1975]. 
Example 2. For an example of a voting rule which satisfies Axioms 1, 2, and $3 a$, but which is not a scoring rule, consider the following. Let $K=\{1,2,3,4\}$, and let $V=\{$ red, yellow, blue $\}$. For any vote distribution $\alpha$, let the normalized distribution be $\hat{\alpha}$ defined by

$$
\hat{\alpha}(\mathrm{v})=\alpha(\mathrm{v}) /\left(\sum_{\mathrm{w} \in \mathrm{V}} \alpha(\mathrm{w})\right), \quad \forall \mathrm{v} \in \mathrm{V} .
$$

We may denote any normalized distribution by an ordered triple of numbers, using the ordering

$$
\hat{\alpha}=(\hat{\alpha}(\text { red }), \hat{\alpha}(\text { yellow }), \hat{\alpha}(\text { blue })) .
$$

Now, we specify that alternative 1 is in the choice set $F(\alpha)$ iff the normalized distribution $\hat{\alpha}$ is in the convex hull of

$$
\{(1,0,0),(5 / 8,2 / 8,1 / 8),(1 / 8,5 / 8,2 / 8),(0,1,0)\}
$$

alternative 2 is in the choice set $F(\alpha)$ iff the normalized distribution $\hat{\alpha}$ is in the convex hull of

$$
\{(0,1,0),(1 / 8,5 / 8,2 / 8),(2 / 8,1 / 8,5 / 8),(0,0,1)\}
$$

alternative 3 is in the choice set $F(\alpha)$ iff the normalized distribution $\hat{\alpha}$ is in the convex hull of

$$
\{(0,0,1),(2 / 8,1 / 8,5 / 8),(5 / 8,2 / 8,1 / 8),(1,0,0)\}
$$

and alternative 4 is in the choice set $F(\alpha)$ iff the normalized distribution $\hat{\alpha}$ is in the convex hull of

$$
\{(5 / 8,2 / 8,1 / 8),(1 / 8,5 / 8,2 / 8),(2 / 8,1 / 8,5 / 8)\} \text {. }
$$

\section{[INSERT FIGURE 1 ABOUT HERE]}

For any scoring rule, if one alternative were eliminated, then we could still apply the same $S_{j}$ functions to define a new scoring rule among the remaining alternatives. The domain where each remaining alternative wins under this new rule would be a convex set of distributions that includes all 
distributions for which this alternative won under the original rule, and that excludes all distributions for which this alternative was beaten by some other remaining alternative under the original rule.

However, when we try to eliminate alternative 4 in this example, we cannot divide alternative $4^{\prime} \mathrm{s}$ domain among alternatives 1,2 , and 3 without making at least one alternative's domain nonconvex. Extending the $(1,2)$ and $(1,3)$ boundaries, for example, we find that alternative 1 cannot win when the normalized distribution is outside of the convex hull of

$$
\{(1,0,0),(0,1,0),(1 / 7,4 / 7,2 / 7)\},
$$

This convex hull excludes the normalized distribution $(1 / 3,1 / 3,1 / 3)$. Thus, in the new voting rule without alternative 4 , alternative 1 could not win when the three kinds of ballot are equally represented, and a symmetric argument shows that alternatives 2 and 3 also could not win then. Thus, this voting rule cannot be represented as a scoring rule.

Bilateral balance functions which satisfy Proposition 3 for this voting rule are

$$
\begin{aligned}
& \mathrm{s}_{12}(\text { red })=-2, \quad \mathrm{~s}_{12}(\text { yellow })=0, \quad \mathrm{~s}_{12}(\text { blue })=1, \\
& s_{23}(\text { red })=1, \quad s_{23}(\text { yellow })=-2, \quad s_{23}(\text { blue })=0, \\
& s_{13}(\text { red })=0, \quad s_{13}(\text { yellow })=-1, \quad s_{13}(\text { blue })=2 \text {, } \\
& \mathrm{s}_{14}(\text { red })=-1, \quad \mathrm{~s}_{14}(\text { yellow })=-9, \quad \mathrm{~s}_{14}(\text { blue })=23 \text {, } \\
& s_{24}(\text { red })=23, s_{24}(\text { yellow })=-1, s_{24}(\text { blue })=-9 \text {, } \\
& s_{34}(\text { red })=-9, \quad s_{34}(\text { yellow })=23, \quad s_{34}(\text { blue })=-1 .
\end{aligned}
$$

Outside of the triangle where 4 wins, the winner is determined by the bilateral balances among alternatives 1, 2, and 3. Alternative 2 has a nonnegative balance against alternative 1 iff there are at least twice as many blue votes as red votes in the distribution. Alternative 3 has a nonnegative balance 
against alternative 2 iff there are at least twice as many red votes as yellow. Alternative 1 has a nonnegative balance against alternative 3 iff there are at least twice as many yellow votes as blue votes. So when the normalized distribution is near $(1 / 3,1 / 3,1 / 3)$, the bilateral balance functions specify that alternative 2 loses against 1 , alternative 1 loses against 3 , and alternative 3 loses against 2. This intransitivity is not a problem, however, because alternative 4 wins in this central region.

This voting rule, with four alternatives but only three possible votes, obviously violates neutrality. It also violates Axiom $3 \mathrm{~b}$, because there is no distribution for which the three alternatives $\{1,2,3\}$ are al1 in the choice set.

For many applications, the neutrality axiom is quite reasonable. However, neutrality is obviously not a necessary condition for scoring rules, as we show in the next example.

Example 3. Consider a scoring rule in which there are three alternatives and three possible votes, so we may let $K=\{1,2,3\}$ and $V=\{1,2,3\}$, and the scoring functions $S_{i}(v)$ are

$$
\begin{aligned}
& s_{1}(1)=1, \quad S_{2}(1)=0, \quad s_{3}(1)=0, \\
& s_{1}(2)=0, \quad S_{2}(2)=\sqrt{2}, \quad S_{3}(2)=0, \\
& s_{1}(3)=0, \quad S_{2}(3)=0, \quad s_{3}(3)=\sqrt{ } 3 .
\end{aligned}
$$

That is, a voter can vote for any alternative in $\mathrm{K}$, but votes for alternatives 2 and 3 are counted more than votes for alternative 1 . This scoring rule is obviously not neutral. A tie between alternatives 1 and 2 could occur only if the number of voters for 1 was greater than the number of voters for 2 by a multiplicative factor equal to the square root of 2 , which is impossible when the number of each kind of vote is an integer. So this voting rule violates 
both neutrality and the strong range condition, but it is a scoring rule.

Examples 2 and 3 suggest that we should look for ways of strengthening Axiom 1 or 1 a so that scoring rules can be derived without neutrality or the strong range condition. For example, Axiom la (nonreversal) could be strengthened to a kind of transitivity axiom as follows.

Axiom 1b (Transitivity). For any distributions $\alpha, \beta, \gamma_{1}, \gamma_{2}$, and $\gamma_{3}$ in $\mathrm{Z}_{+}^{\mathrm{V}}$, and for any alternatives $\mathrm{h}, i$, and $j$ in $\mathrm{k}$, if $\mathrm{h} \in \mathrm{F}\left(\gamma_{1}+\alpha\right)$, i $\in \mathrm{F}\left(\gamma_{1}+\beta\right)$, $i \in F\left(\gamma_{2}+\alpha\right), j \in F\left(\gamma_{2}+\beta\right), j \in F\left(\gamma_{3}+\alpha\right)$, and $h \in F\left(\gamma_{3}+\beta\right)$, then $\{h, i\} \subseteq F\left(\gamma_{1}+\alpha\right) \cap F\left(\gamma_{1}+\beta\right),\{i, j\} \subseteq F\left(\gamma_{2}+\alpha\right) \cap F\left(\gamma_{2}+\beta\right)$, and $\{h, j\} \subseteq F\left(\gamma_{3}+\alpha\right) \cap F\left(\gamma_{3}+\beta\right)$.

Any scoring rule must satisfy this transitivity condition, and it is violated by the nonscoring rule in Example 2. For example, let

$$
\begin{aligned}
& \left.\gamma_{1} \text { (red) }=1, \quad \gamma_{1} \text { (yellow }\right)=0, \quad \gamma_{1} \text { (blue) }=100, \\
& \left.\gamma_{2} \text { (red) }=0, \quad \gamma_{2} \text { (yellow }\right)=100, \quad \gamma_{2} \text { (blue) }=1, \\
& \left.\left.\gamma_{3} \text { (red) }=100, \quad \gamma_{3} \text { (yellow }\right)=1, \quad \gamma_{3} \text { (blue }\right)=0, \\
& \alpha(\text { red })=0, \quad \alpha \text { (yellow })=0, \quad \alpha \text { (blue) }=0 . \\
& \beta(\text { red })=3, \quad \beta \text { (yellow })=3, \quad \beta \text { (blue })=3 .
\end{aligned}
$$

Then we get the following violation of this transitivity axiom:

$$
\begin{aligned}
& F\left(\gamma_{1}+\alpha\right)=\{3\}, \quad F\left(\gamma_{1}+\beta\right)=\{2\}, \\
& F\left(\gamma_{2}+\alpha\right)=\{2\}, \quad F\left(\gamma_{2}+\beta\right)=\{1\}, \\
& F\left(\gamma_{3}+\alpha\right)=\{1\}, \quad F\left(\gamma_{3}+\beta\right)=\{3\} .
\end{aligned}
$$

At this time, however, I do not know whether this transitivity axiom is sufficient generally to derive a scoring-rule representation without neutrality. 


\section{REFERENCES}

H. Moulin [1988], Axioms of Cooperative Decision Making, Cambridge University Press.

J. Smith [1973], "Aggregation of preferences with a variable electorate," Econometrica 41:1027-1041.

H. P. Young [1975], "Social choice scoring functions," SIAM Journal of Applied Mathematics $28: 824-838$. 


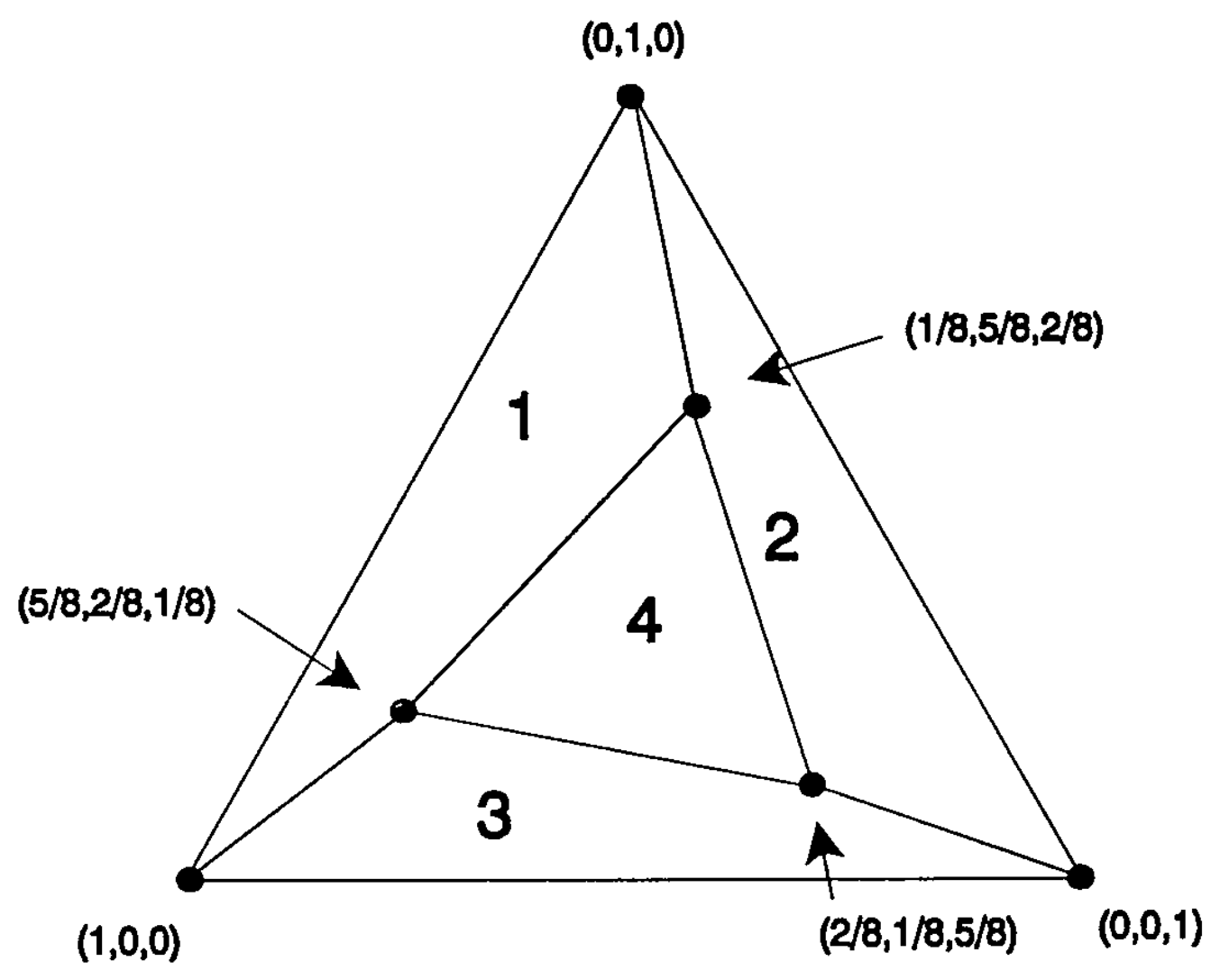

Figure 1. Normalized distributions where each alternative wins, in Example 2 